\title{
Linear Position-Based Visual Predictive Control
}

\author{
M. M. H. Fallah (Student), and F. Janabi-Sharifi \\ Mechanical and Industrial Engineering \\ Department, Ryerson University, \\ Toronto, Ontario, Canada M5B 2K3 \\ Emails: mostafa.mohammad@ryerson.ca, fsharifi@ryerson.ca
}

\begin{abstract}
Although position-based visual servoing (PBVS) scheme guarantees the global asymptotic stability and unlike the image-based visual servoing (IBVS), some issues such as image singularities and camera retreat problems do not emerge within the control procedure but, its sensitivity to camera calibration errors must be addressed. This paper presents a novel positionbased visual predictive control (PVPC) method based on the internal model control (IMC) scheme to not only overcome the issues caused by camera calibration errors, but also to handle the available constraints in the visual servoing procedure. In addition, the optimized control signal puts less pressure on the actuators while the camera goes through the smoother spatial trajectory. In order to verify the functionality and efficiency of the proposed approach, some simulation results are presented .

Index Terms-Visual servoing, Model predictive control, Internal model control, Robotics
\end{abstract}

\section{INTRODUCTION}

Visual servo control (VS) employs the feedback information provided by a visual sensor to control the motion of a robot [1]. There are two classic approaches in VS based on their feedback signals: image-based visual servoing (IBVS) and position-based visual servoing (PBVS). In IBVS, the error is generated directly from image features and mapped consequently to actuators commands. While in PBVS, a geometric model of the object plus known camera intrinsic parameters are used to reconstruct the 3-D pose of the object relative to the camera and to calculate the error and associated control signal to reach the reference pose.

In IBVS, the on-line calculation of the image Jacobian is required, that is a matrix depending on the relative distance between the object and camera which may be difficult to evaluate. In addition, this control method poses problems such as image local minima and/or singularities and camera retreat. On the contrary, the principal advantage of using PBVS the chance of defining tasks in a standard Cartesian frame but, in this case, the control law depends on the camera calibration parameters, and can be widely affected by calibration errors [2]. On the other side, none of the aforementioned approaches could easily deal with available constraints such as the joint limits or the visibility constraints.

Among the various investigated methods reported in the literature to deal with available constraints, the model predictive control (MPC) has brilliant functionality and is a effective tool in constraints handling and finding the optimized control signal [3], [4]. To date, a few works addressed a successful combination of VS problems with MPC framework, often using an error similar to IBVS approach. For example, in [5] and [6] the IBVS-based MPC is formulated to control the motion of an instrument equipped with an ultrasound camera. The convergence of the camera's pose to final reference one has been guaranteed and simultaneously available constraints have been handled, too. In [7] and [8], the visual servoing task is formulated into a nonlinear optimization problem in the image plane utilizing two distinct predictive models, a local model based on the image Jacobian and a non-linear global model. Although the aforementioned MPC methods in [7] and [8] could facilitate the integration of constraints into control solution but, by using the local model based on interaction matrix within MPC framework, there is no direct control on camera's Cartesian trajectory and this may cause the system failure in some scenarios such as 180 deg pure rotation around the camera's optical axis. Also, similar to the previous image-based MPC methods, this approach will suffer from some of the shortcomings of IBVS, including image local minima and singularities. On the other side, forecasting over the long prediction horizon can be time consuming using nonlinear models.

This paper contributes by proposing a position-based predictive control method to enable constraints handling within VS procedure while avoiding some of the mentioned issues with IBVS. On the other side, to overcome the issue caused by the non-linearity of the PBVS, similar to [7] and [8], a linear method based on the the position-based interaction matrix [9] has been used. In addition, by employing the internal model control proposed in [10], the sensitivity of the position-based approach to calibration errors could be eliminated, too.

This paper is organized as follows. Section II details the proposed PVPC approach theory. The stability of the presented control scheme has been investigated in section III. The advantages of developed PVPC in comparison with IVPC, and its functionality and robustness in presence of camera calibration errors are demonstrated in section IV. Finally, conclusions are given in the last section.

\section{Linear Position-Based Visual Predictive CONTROL (PVPC)}

The linear position-based visual predictive (PVPC) control is designed based on model predictive control (MPC) method. PVPC employs a model of the system to not only optimize the 3-D trajectory of camera in the space, but also to handle 


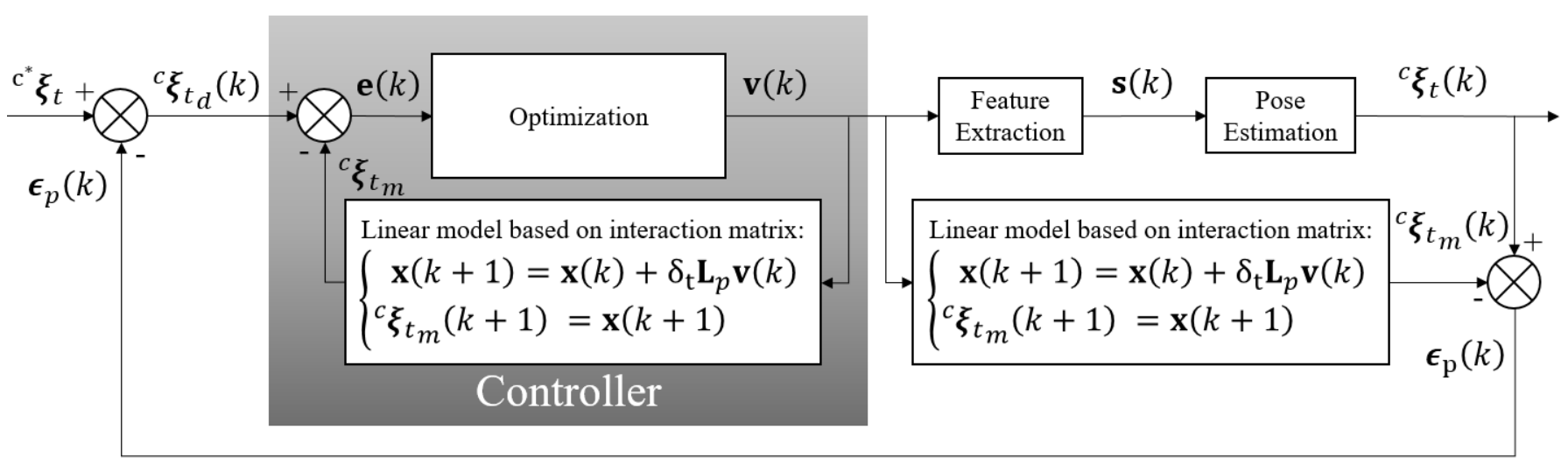

Fig. 1: Block diagram of linear position-based visual predictive control (PVPC).

available constraints. The associated block diagram of proposed control scheme based on internal model control (IMC) [10] is shown in Fig. 1. In this block diagram, $\mathbf{v}(k), \mathbf{x}(k)$, and ${ }^{c} \boldsymbol{\xi}_{t}(k)$ are the control signal or the system input, the system state, and the system output, respectively. Similar to any other MPC scheme, four essential ingredients including a reference signal, a plant's predictive model, a cost function to be minimized over the prediction horizon, and an efficient optimization method to find the optimal control signal are necessary.

In the proposed scheme, there is a static or dynamic reference signal, ${ }^{*} \boldsymbol{\xi}_{t}(k)$ which is the 3-D relative target pose with respect to reference camera frame (or the robot end-effector frame in the robotics applications). The error signal $\boldsymbol{\epsilon}_{p}(k)$ is the subtraction of the model predicted vector, ${ }^{c} \boldsymbol{\xi}_{t_{m}}(k)$, from the measured one, ${ }^{c} \boldsymbol{\xi}_{t}(k)$. This signal includes all disturbances and modeling errors and is utilized to calculate the desired pose signal, ${ }^{c} \boldsymbol{\xi}_{t_{d}}(k)$ to be tracked by the predicted one. Indeed, we have [7], [8].

$$
\begin{gathered}
{ }^{c} \boldsymbol{\xi}_{t_{d}}(k)={ }^{c^{*}} \boldsymbol{\xi}_{t}(k)-\boldsymbol{\epsilon}_{p}(k)= \\
{ }^{c^{*}} \boldsymbol{\xi}_{t}(k)-\left({ }^{c} \boldsymbol{\xi}_{t}(k)-{ }^{c} \boldsymbol{\xi}_{t_{m}}(k)\right),
\end{gathered}
$$

and consequently

$$
{ }^{c} \boldsymbol{\xi}_{t d}(k)-{ }^{c} \boldsymbol{\xi}_{t_{m}}(k)={ }^{c} \boldsymbol{\xi}_{t}(k)-{ }^{c} \boldsymbol{\xi}_{t}(k) .
$$

All MPC schemes need a predictive model of plant to anticipate the value of the system output based on the current state and implemented control signal. In classic positionbased visual servoing of a robot, the model of the robotic system, combined with a camera's model and nonlinear pose estimation procedure, all, increase the the nonlinearity level of the predictive model. Consequently, the prediction time will be increased drastically. To solve this issue, Allibert et al. [7], [8] developed a linear image-based predictive model based on the image Jacobian matrix. Similarly, in this work, by employing the corresponding interaction matrix, a linear position-based model has been developed. In addition, point features are utilized for camera pose estimation.
Considering ${ }^{c} \boldsymbol{\xi}_{t_{m}}(k)=\left({ }^{c} \mathbf{T}_{t m}(k), \theta \mathbf{u}_{m}\right)$ and ${ }^{c} \boldsymbol{\xi}_{t_{d}}(k)=$ $\left({ }^{c} \mathbf{T}_{t d}(k), \mathbf{0}\right)$, position-based interaction matrix, $\mathbf{L}_{p}$, is given by [9]

$$
\mathbf{L}_{p}=\left[\begin{array}{cc}
-\mathbf{I}_{3} & {\left[{ }^{c} \mathbf{T}_{t m}\right]_{\times}} \\
\mathbf{0} & \mathbf{L}_{\theta \mathbf{u}_{m}}
\end{array}\right],
$$

where $\mathbf{I}_{3}$ is the $(3 \times 3)$ identity matrix, $\left[{ }^{c} \mathbf{T}_{t m}\right]_{\times}$is the equivalent $(3 \times 3)$ skew symmetric matrix of ${ }^{c} \mathbf{T}_{t m}$, the translational part of ${ }^{c} \boldsymbol{\xi}_{t_{m}}(k)$, and $\mathbf{L}_{\theta \mathbf{u}_{m}}$ is given by

$$
\mathbf{L}_{\theta \mathbf{u}_{m}}=\mathbf{I}_{3}-\frac{\theta_{m}}{2}\left[\mathbf{u}_{m}\right]_{\times}-\left(1-\frac{\operatorname{sinc} \theta_{m}}{\operatorname{sinc}^{2} \frac{\theta_{m}}{2}}\right)\left[\mathbf{u}_{m}\right]_{\times}^{2},
$$

where $\theta \mathbf{u}_{m}$ is the axis-angle representation of the rotational part of ${ }^{c} \boldsymbol{\xi}_{t_{m}}(k)$ and sinc is the sinus cardinal defined such that $x \operatorname{sinc} x=\sin x$ and $\operatorname{sinc} 0=1$. The dynamic evolution of camera pose is expressed by

$$
{ }^{c} \dot{\boldsymbol{\xi}}_{t_{m}}(k)=\mathbf{L}_{p} \mathbf{v}(k),
$$

where $\mathbf{L}_{p}$ represents the position-based interaction matrix. By utilizing the Newton-Euler method the next approximated target/camera relative pose would be obtained as

$$
\left\{\begin{array}{l}
\mathbf{x}_{m}(k+1)=\mathbf{x}_{m}(k)+\delta_{t} \mathbf{L}_{p} \mathbf{v}(j), \\
{ }^{c} \boldsymbol{\xi}_{t_{m}}(k)=\mathbf{x}_{m}(k),
\end{array}\right.
$$

where $\delta_{t}$ is the discrete time step and $\mathbf{v}(k), \mathbf{x}_{m}(k)$ and ${ }^{c} \boldsymbol{\xi}_{t m}(k)$ are system input, system state, and system output, respectively.

The cost function, $\Upsilon$, is defined as the sum of the quadratic position-based errors over the prediction horizon. In order to obtain the optimal control signal, this scalar function of $\mathbf{v}(k)$, must be minimized. Consequently, the mathematical formulation of proposed control scheme can be expressed as

$$
\min _{\hat{\mathbf{v}} \in \mathbb{R}^{q \times N_{p}}} \Upsilon(\mathbf{v}(k)),
$$

where

$$
\begin{gathered}
\Upsilon(\mathbf{v}(k))= \\
\left.\sum_{j=k+1}^{k+N_{p}}\left\|{ }^{c} \boldsymbol{\xi}_{t_{d}}(j)-{ }^{c} \boldsymbol{\xi}_{t_{m}}(j)\right\|\right|^{\top} \Phi\left\|{ }^{c} \boldsymbol{\xi}_{t_{d}}(j)-{ }^{c} \boldsymbol{\xi}_{t_{m}}(j)\right\|,
\end{gathered}
$$


TABLE I: Camera intrinsic parameters.

\begin{tabular}{llll}
\hline Parameter & Value & Parameter & Value \\
\hline focal length & $8 \mathrm{~mm}$ & pixel size & $(0.01,0.01) \mathrm{mm}$ \\
principal point & $(512,512)$ & number of pixels & $1024 \times 1024$ \\
\hline
\end{tabular}

There is no distortion in the camera model.

where $q, N_{p}$, and $\hat{\mathbf{v}}$ are the size of control signal vector, prediction horizon, and the optimal control sequence, respectively. In addition, $\Phi$ is the weighting matrix to tune the effectiveness of the available terms which is equal to identity matrix in the following simulations.

Regarding to its brilliant ability to solve the nonlinear constrained optimization problems, among different algorithms, a Sequential Quadratic Program (SQP) is utilized in this work. The SQP can be described as a generalized Newton's method and its equivalent for constrained optimization. In SQP, by minimizing a quadratic model of the problem, a step away from the current point will be found.

As it is mentioned before, during the optimization procedure, there are different constraints to be handled which can be expressed as follows

1) Spatial constraints: such as workspace boundaries (or joint limits in robotics applications) and depth of field (the relative depth of target feature in camera frame) as an important visual constraint

$$
{ }^{c} \boldsymbol{\xi}_{t_{\min }} \leq{ }^{c} \boldsymbol{\xi}_{t_{m}}(k) \leq{ }^{c} \boldsymbol{\xi}_{t_{\text {max }}} .
$$

2) Control signal constraints: such as camera's velocity boundaries (or actuators' saturation limits in robotics applications)

$$
\mathbf{v}_{\min } \leq \mathbf{v}(k) \leq \mathbf{v}_{\max } .
$$

3) Terminal equality constraint: Although PBVS itself is globally asymptotic stable, but meeting this constraint guarantees the closed-loop stability of the proposed MPC scheme

$$
{ }^{c} \boldsymbol{\xi}_{t_{m}}\left(k+N_{p}+1\right)-{ }^{c} \boldsymbol{\xi}_{t d}\left(k+N_{p}+1\right)=\mathbf{0} .
$$

\section{Stability ANALysis}

As it is proven in [9], PBVS itself is globally asymptotic stable. In addition, consider that the cost function in a MPC system is defined as

$$
\Upsilon(\mathbf{v}(k))=\sum_{j=k+1}^{k+N_{p}} h(\mathbf{x}, \mathbf{v}(k))+T\left(\mathbf{x}\left(k+N_{p}+1\right)\right),
$$

where $h(\mathbf{x}, \mathbf{v}(k))$ and $T\left(\mathbf{x}\left(k+N_{p}+1\right)\right.$ are the cost value at time instant, $k$, and terminal cost respectively. In addition, let $\eta^{*}($.$) denote the change in \eta($.$) as the system state changes$ from $\mathbf{x}(k)$ to $\mathbf{x}(k+1)$. The closed-loop asymptotic stability of the control system will be guaranteed if the following four axioms are satisfied [11]:
A1. $X_{f} \subset \mathbb{X}, X_{f}$ closed, and $\mathbf{0} \in X_{f}$, where $X_{f}$ is the terminal constraint and $\mathbb{X}$ is a closed and convex subset of $\mathbb{R}^{n}$ including all of the available system states and $n$ is the length of state vector, $\mathbf{x}$.

$\triangleright$ In this work, the origin is shifted from $\mathbf{0}$ to ${ }^{c} \boldsymbol{\xi}_{t_{d}}$ and $X_{f}=\{\boldsymbol{0}\}$. Accordingly, the terminal constraint (11) guarantees the sanctification of $\mathbf{A 1}$.

A2. $\kappa(\mathbf{x}, k) \subset \mathbb{V}, \forall \mathbf{x} \in X_{f}$, where $\kappa$ defines an implicit model predictive control law, $\mathbf{x}$ is the system state at time instant $k, \mathbb{V}$ is a convex, compact subset of $\mathbb{R}^{q}$ including all of acceptable system inputs, and $q$ is the length of control signal, $\mathbf{v}$. In addition, $\kappa(\mathbf{x}, k):=\mathbf{v}(k,(\mathbf{x}, k))$.

$\triangleright$ In this paper, $\kappa$ obtained from PBVS, implicitly, and at the origin, where $\left({ }^{c} \boldsymbol{\xi}_{t m}={ }^{c} \boldsymbol{\xi}_{t_{d}}\right), \kappa:=\mathbf{0} \subset \mathbb{V}$. Accordingly, $\mathbf{A 2}$ is satisfied, too.

A3. $g(\mathbf{x}, \kappa(\mathbf{x})) \in X_{f}, \forall \mathbf{x} \in X_{f}$, where $g(\mathbf{x}, \kappa(\mathbf{x}))$ represents the system predictive model $(\mathbf{x}(k+1)=$ $g(\mathbf{x}(k), \kappa(\mathbf{x}(k))))$.

$\triangleright$ Here, $\kappa:=\mathbf{0}, \forall \mathbf{x} \in X_{f}$. So, this axiom follows from the fact that $g\left({ }^{c} \boldsymbol{\xi}_{t d}, \kappa(\mathbf{0})\right)=g\left({ }^{c} \boldsymbol{\xi}_{t d}, \mathbf{0}\right)=\mathbf{0} \in X_{f}$.

A4. $[\stackrel{*}{T}+h](\mathbf{x}, \kappa(\mathbf{x})) \leq 0, \forall \mathbf{x} \in X_{f}$.

$\triangleright$ in this paper,

$$
\left.[\stackrel{*}{T}+h]\left({ }^{c} \boldsymbol{\xi}_{t_{d}}, \kappa(\mathbf{0})\right)=[\stackrel{*}{T}+h]\left({ }^{c} \boldsymbol{\xi}_{t_{d}}, \mathbf{0}\right)\right)=0
$$

and the satisfaction of the forth axiom will be ensured.

Therefore, by enforcing the system to meet (11), the proposed control system will be globally asymptotically stable.

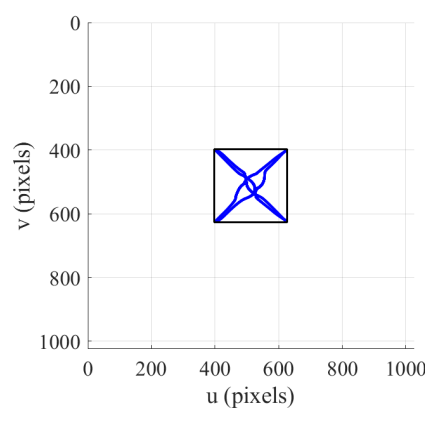

(a) Image plane

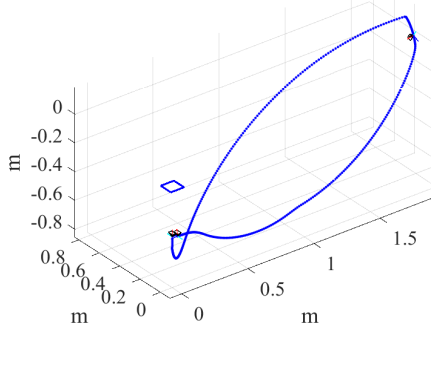

(b) Camera 3-D trajectory
Fig. 2: Failure of the image-based VPC with $\mathbf{L}_{c}$ and $N_{p}=30$ in Chaumette Conundrum due to the camera retreat problem.

\section{Simulation Results}

\section{A. Position-Based VPC (PVPC) vs Image-Based VPC (IVPC)}

In order to compare the effectiveness of position-based VPC with image-based one, some scenarios have been designed and simulated. In these simulations, it is assumed that camera can fly freely in the 3-D space with 6 DOF. In addition, the discrete sampling time step, $\delta_{t}$, is $0.4 \mathrm{~ms}$. Table I includes the intrinsic parameters of the simulated camera used in associated subroutine of MATLAB ${ }^{\circledR}$. 


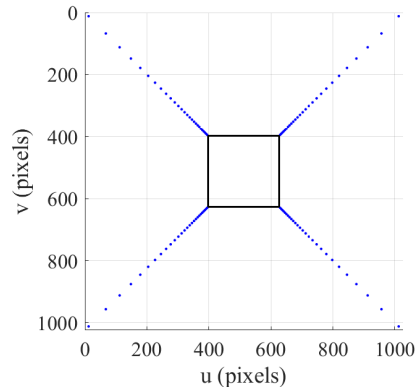

(a) Image plane

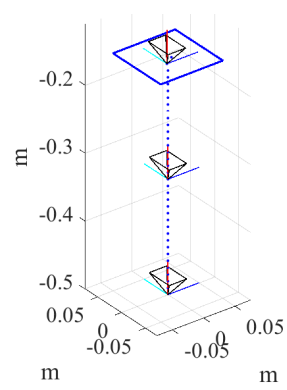

(b) Camera 3-D trajectory
Fig. 3: Failure of the image-based VPC with $\mathbf{L}_{d}$ and $N_{p}=30$ in Chaumette Conundrum due to the camera advance problem.

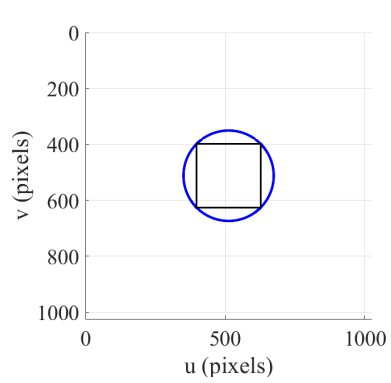

(a) Image plane

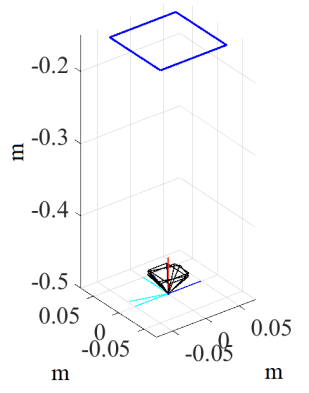

(b) Camera 3-D trajectory
Fig. 4: Linear position-based VPC with $N_{p}=30$ converges to desired configuration successfully in Chaumette Conundrum.

In these three scenarios, shown in Fig. 2 to Fig. 4, the effectiveness of image-based VPC schemes with different interaction matrices have been compared with the proposed scheme under 180 deg pure rotation around the cameras optical axis or Chaumette Conundrum. In mentioned scenarios, the target comprises four vertices of a square with $10 \mathrm{~cm}$ length which is located on a plane perpendicular to the camera optical axis, and $30 \mathrm{~cm}$ far from its origin.

As it is shown in Figs. 2 and 3, both of the image-based VPC schemes with either $\mathbf{L}_{c}{ }^{1}$ or $\mathbf{L}_{d}{ }^{2}$ fail due to significant camera retreat and advance problems, respectively. Even by increasing the prediction horizon, $N_{p}$, from 30 to 300 , this problem remains. In addition, the larger horizon, will be computationally non-affordable to be employed by control systems. On the other hand, due to having direct control on the camera pose, the proposed position-based VPC scheme performs 180 deg pure rotation task successfully, as shown in Fig. 4.

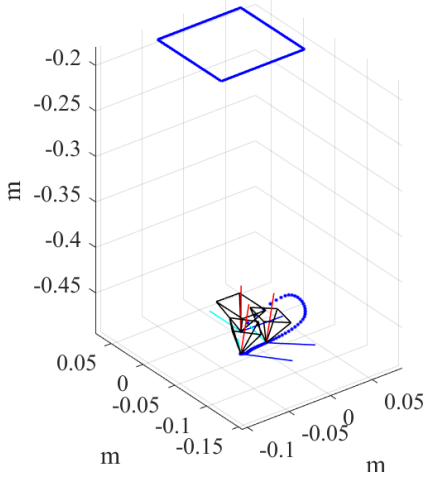

(a) Camera trajectory with $N_{p}=10$ from the first point of view

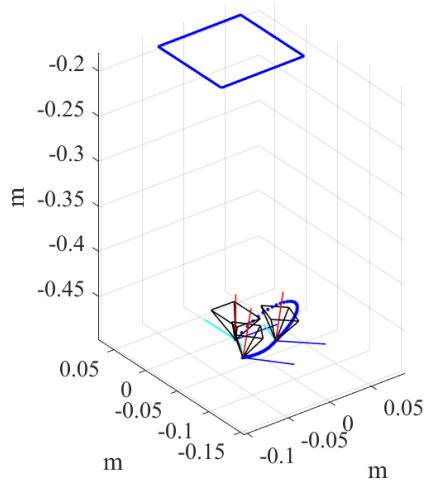

(c) Camera trajectory with $N_{p}=20$ from the first point of view

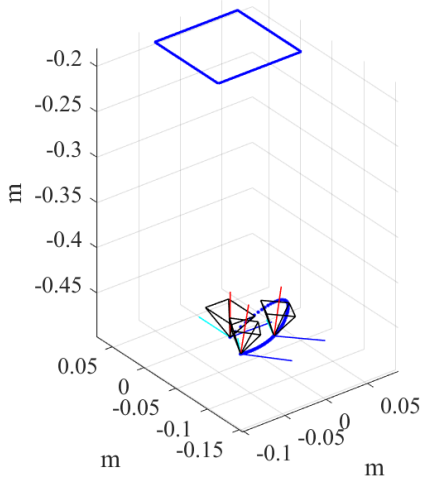

(e) Camera trajectory with $N_{p}=30$ from the first point of view

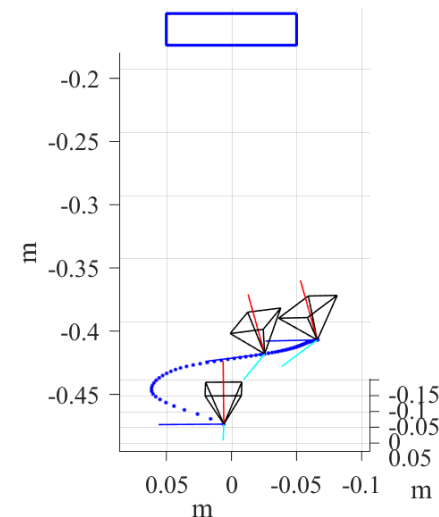

(b) Camera trajectory with $N_{p}=10$ from the second point of view

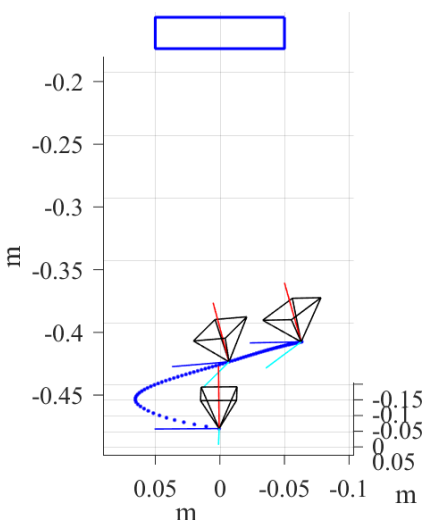

(d) Camera trajectory with $N_{p}=20$ from the second point of view

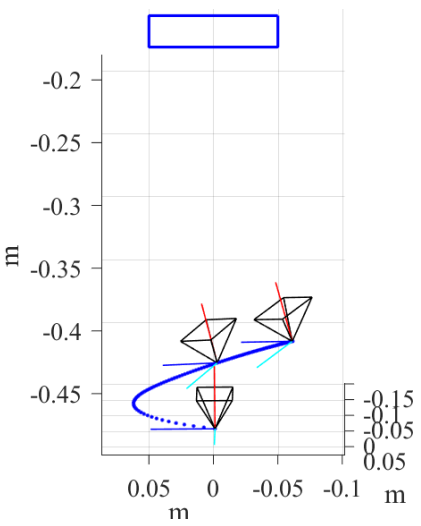

(f) Camera trajectory with $N_{p}=30$ from the second point of view

Fig. 5: Reduction of unnecessary camera motion by increasing the prediction horizon, $N_{p}$. 


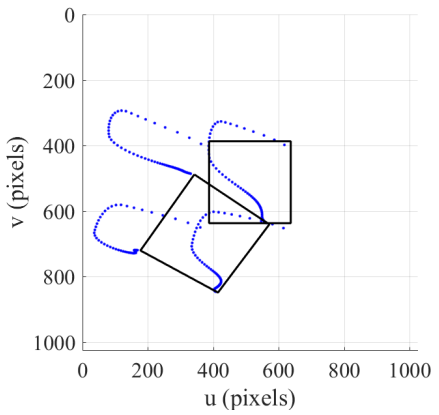

(a) Image plane with $N_{p}=10$

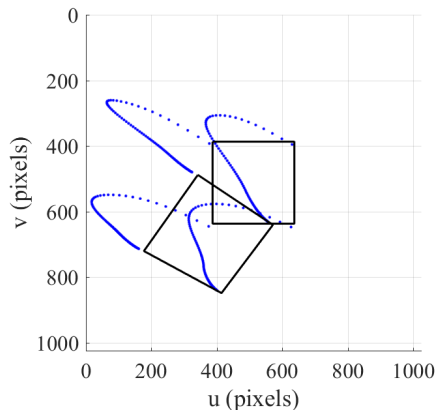

(b) Image plane with $N_{p}=20$

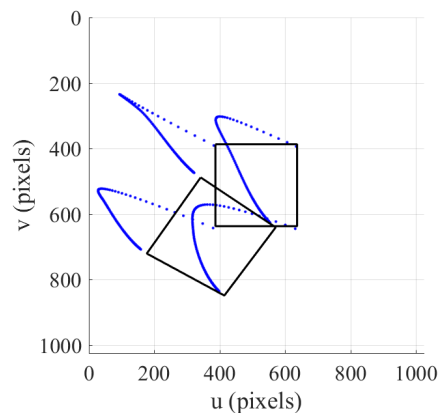

(c) Image plane with $N_{p}=30$

Fig. 6: Smoother visual feature trajectories by increasing the prediction horizon, $N_{p}$.

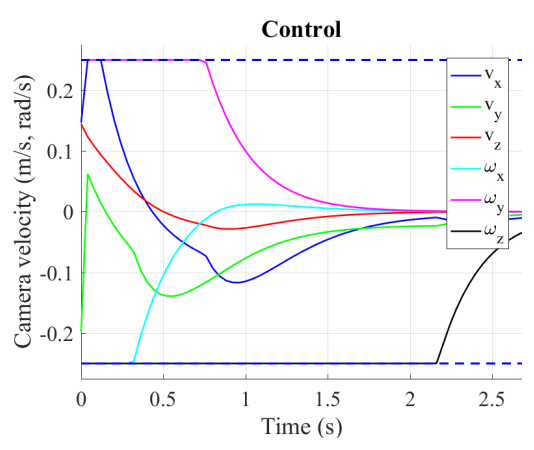

(a) Control signal with $N_{p}=10$

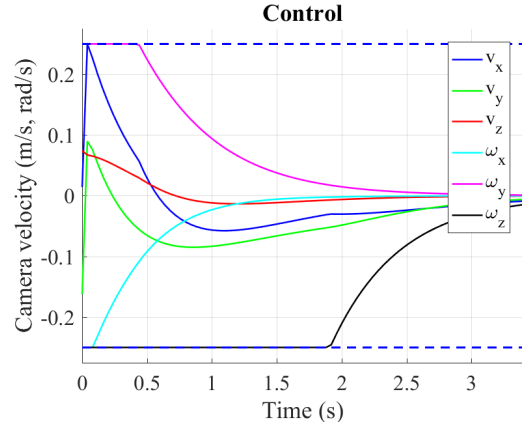

(b) Control signal with $N_{p}=20$

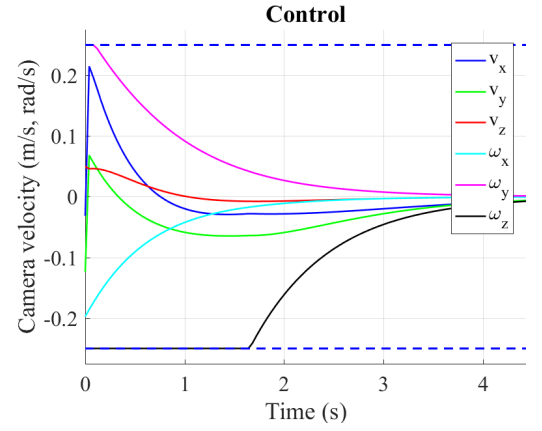

(c) Control signal with $N_{p}=30$

Fig. 7: Optimum control signal wrt. available camera velocity limits.

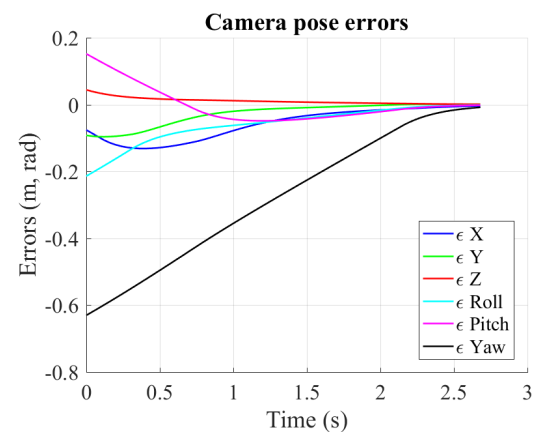

(a) Pose errors with $N_{p}=10$

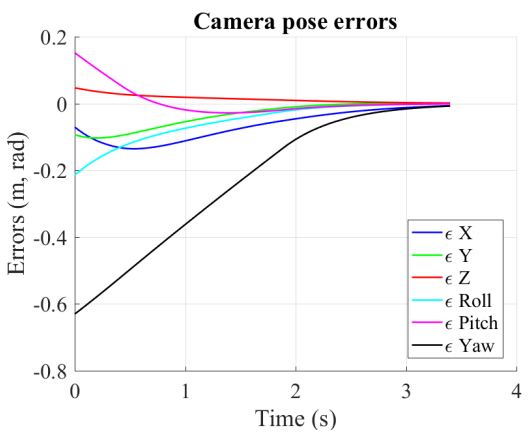

(b) Pose errors with $N_{p}=20$

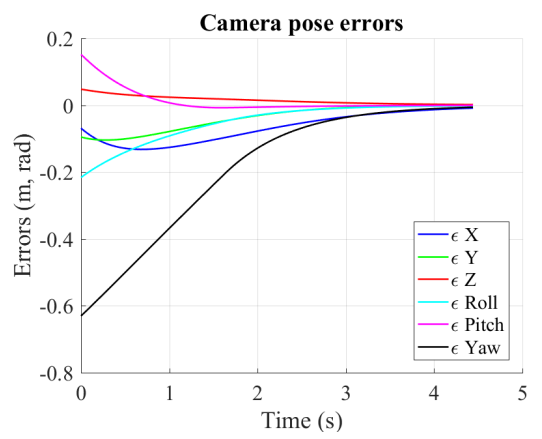

(c) Pose errors with $N_{p}=30$

Fig. 8: 3-D pose errors gradually vanish by utilizing the proposed control structure.
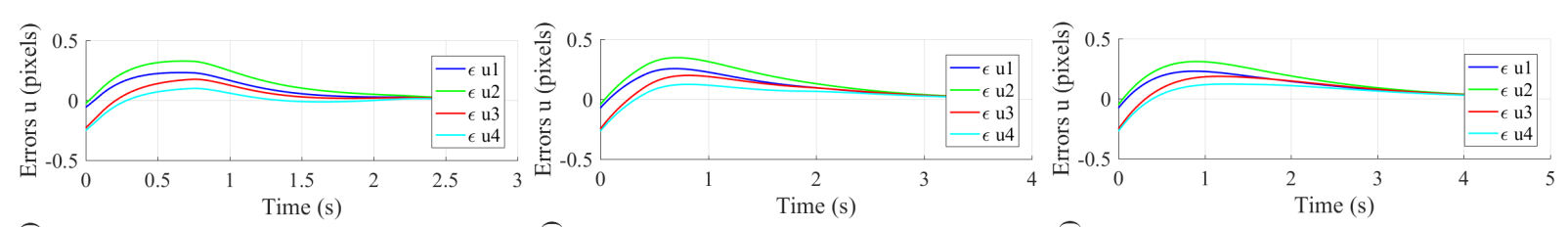

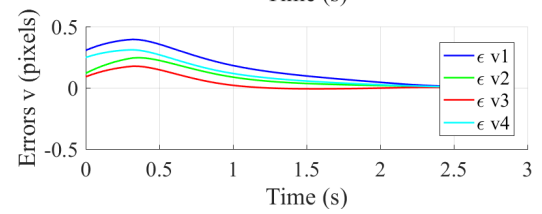

(a) Visual errors with $N_{p}=10$

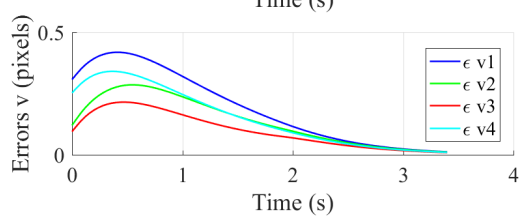

(b) Visual errors with $N_{p}=20$

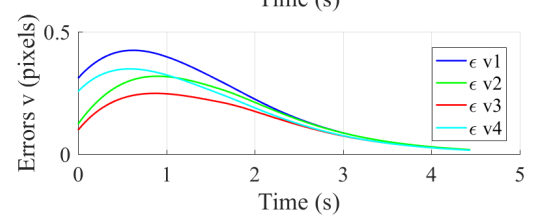

(c) Visual errors with $N_{p}=30$

Fig. 9: Eliminating 2-D visual errors by implementing the PVPC. 


\section{B. Functionality of the PVPC}

Objective of the next simulation set is to analyze the functionality of the proposed position-based visual predictive control scheme and its ability to handle available spatial and operational constraints. In these scenarios, the target includes four vertices of a square with the length of $10 \mathrm{~cm}$ located on a plane, perpendicular to the camera's optical axis, and $32 \mathrm{~cm}$ far from its origin. In addition, while the initial position of the camera with respect to reference coordinate frame is $(0,0,-0.5) \mathrm{m}$, it is assigned to be translated by another $-0.07 \mathrm{~m},-0.1 \mathrm{~m}$, and $0.05 \mathrm{~m}$ along the $x, y$, and $z$-axes respectively, and is rotated by $-5 \mathrm{deg}, 15 \mathrm{deg}$ and $35 \mathrm{deg}$ around the aforementioned global axes to reach the final reference pose as shown in Fig. 5. The initial pixel-wise coordinates of visual features are then $(387,387),(387,637)$, $(637,637)$, and $(637,387)$, respectively, and the corresponding reference coordinates are $(342,488),(177,720),(414,849)$, and $(571,641)$, as demonstrated in Fig. 6.

The relative pose of target with respect to camera has not to exceed the predefined 3-D constraints which are

$$
\left[\begin{array}{c}
-0.2 \\
-0.2 \\
-0.6 \\
-\pi \\
-\pi \\
-\pi
\end{array}\right] \leq^{c} \boldsymbol{\xi}_{t_{m}} \leq\left[\begin{array}{c}
0.2 \\
0.2 \\
-0.1 \\
\pi \\
\pi \\
\pi
\end{array}\right],
$$

where, the first three elements of each vector are expressed in $\mathrm{m}$ and the last three ones are in rad. In addition, there is a camera velocity constraint to be fulfilled during the optimization. In this scenario set, we consider $-0.25 \mathrm{~m} / \mathrm{s}(\mathrm{rad} / \mathrm{s})$ and $0.25 \mathrm{~m} / \mathrm{s}(\mathrm{rad} / \mathrm{s})$ as the lower and the upper bounds of each translational (rotational) element of the control signal, $\mathbf{v}(k)$, respectively. These boundary limits are shown by dashed blue lines in Fig. 7.

As it is clear in Fig. 5 and Fig. 7, all of the spatial and control signal elements stay in the predefined permitted ranges during the camera's travel from initial to reference configuration. The 3-D camera trajectory get smoother by increasing the prediction horizon, $N_{p}$, and the unnecessary camera motions are gradually eliminated. In addition, although the final system convergence time is increased (Fig. 8 and Fig. 9), but by increasing $N_{p}$, the optimal control signal takes more distance from its boundary limits which means having less pressure on the system actuators (Fig. 7).

\section{Robustness of the PVPC}

1) In the presence of the additive noise: The objective of the following simulation sets is to test the robustness of the PVPC in presence of camera calibration errors. Indeed, to illustrate the inaccuracy in pose estimation procedure, a white noise with amplitude of $0.02 \mathrm{~m}$ (or rad) and uniform

\footnotetext{
${ }^{1}$ The interaction matrix in which the current coordinate of image feature and its current 3-D depth with respect to camera frame are used [7].

${ }^{2}$ The interaction matrix in which the reference coordinate of image feature and its reference 3-D depth with respect to camera frame are used [7].
}

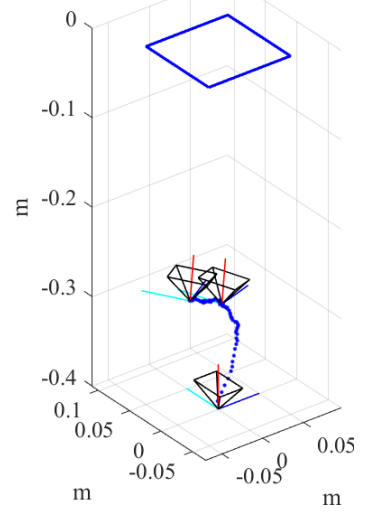

(a) Camera trajectory from the first point of view

(c) Image features trajectories

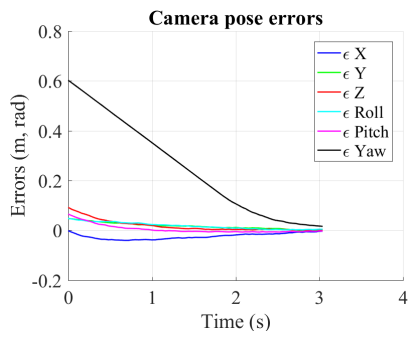

(e) Camera pose errors

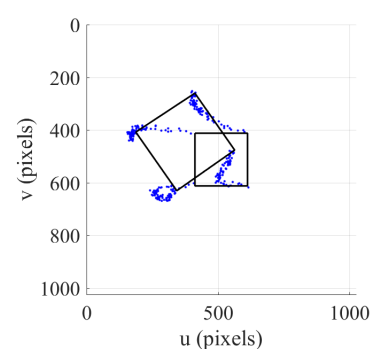

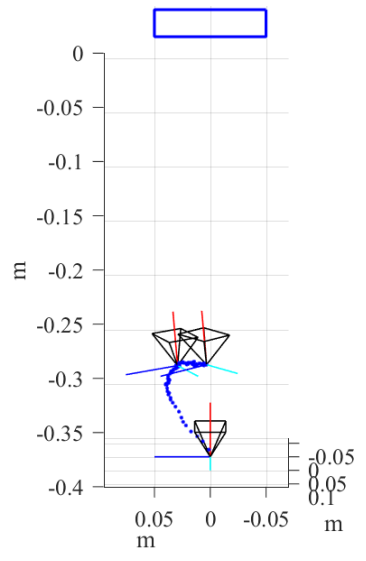

(b) Camera trajectory from the second point of view

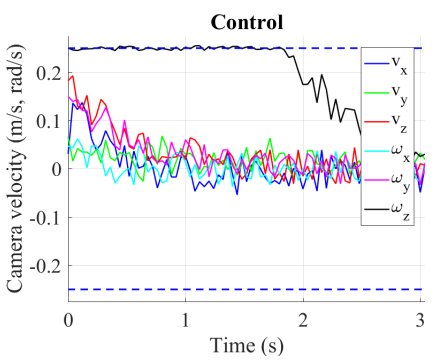

(d) Optimum control signal

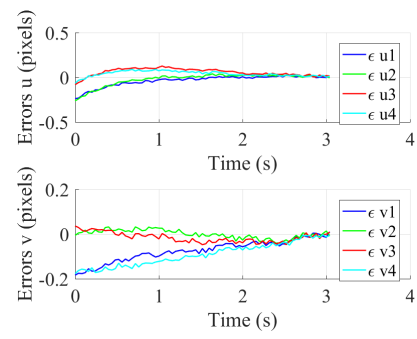

(f) Image features errors
Fig. 10: Robustness of the proposed linear PVPC scheme in presence of the additive noises $\left(N_{p}=20\right)$.

distribution is added to the final estimated pose. In addition to demonstrate the inaccuracy in the image processing, another additive white noise with amplitude equal to 10 pixels is added to the camera 2-D measurement which itself affects the final estimated pose, too. Finally, to demonstrate the effect of errors in robot actuations, a white noise with amplitude equal to 2 percent of the calculated control signal is added to it. As it is shown in Fig. 10, due to having the same structure as the internal model control proposed in [10], the system is robust to all of the aforementioned noises.

2) In the presence of the multiplicative noise: In the last simulation set, the system is polluted with the same noises as previous scenario but, instead the additive noise, the calibration error is simulated by a multiplicative white noise with amplitude equal to 5 percent of the estimated pose. Similarly, the internal model control guarantees the system robustness, as shown in Fig. 11. 


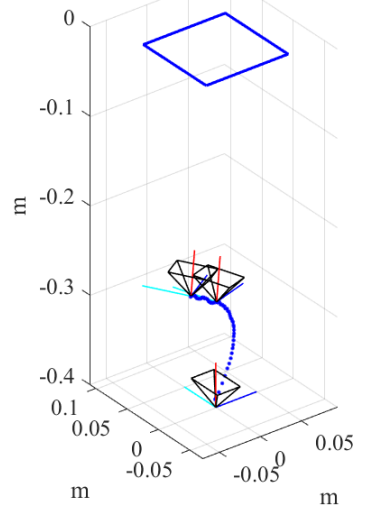

(a) Camera trajectory from the first point of view

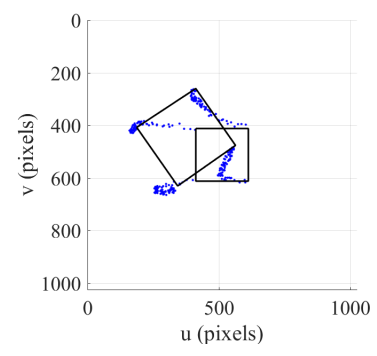

(c) Image features trajectories

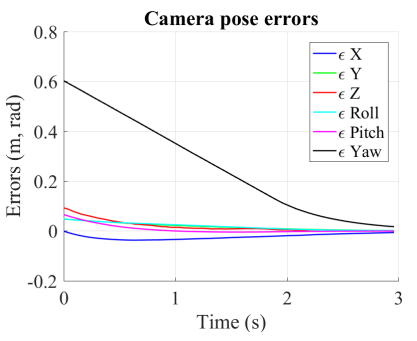

(e) Camera pose errors

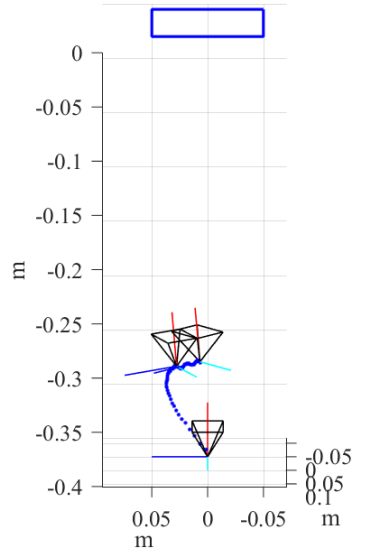

(b) Camera trajectory from the second point of view

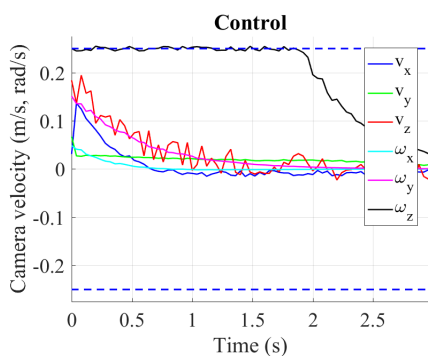

(d) Optimum control signal

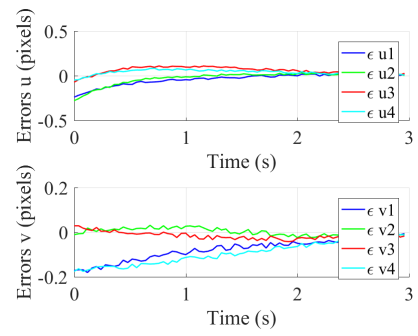

(f) Image features errors
Fig. 11: Robustness of the proposed linear PVPC scheme in presence of the multiplicative noises $\left(N_{p}=20\right)$.

\section{CONClusion}

In this paper, the classic position-based visual servoing (PBVS) approach has been combined with internal model control (IMC) scheme. The novel proposed structure called position-based visual predictive control (PVPC) not only decreases the sensitivity to camera calibration errors, but also handles some of the physical constraints such as joints and actuators limits.

The advantages of proposed scheme in comparison with equivalent image-based approach (IVPC) were simulated in different scenarios. It was shown that, unlike the image-based visual predictive control, there is no issue in scenarios with significant pure rotation around the camera's optical axis.

Furthermore, the functionality and robustness of the proposed method have been examined in three simulation sets. As it was shown in figures, increasing prediction horizon, $N_{p}$, led to smoother camera spatial trajectory and lower pressure on the system actuators.

In addition, it is shown that PVPC is somehow robust to the camera calibration errors where the PBVS control scheme fails due to its high level of sensitivity.

\section{REFERENCES}

[1] P. I. Corke, Visual Control of Robots: High-Performance Visual Servoing, Taunton, UK: Research Studies Press, 1996.

[2] F. Janabi-Sharifi, "Visual Servoing: Theory and Applications," OptoMechatronic Systems Handbook, Cleveland, OH, USA: CRC Press, 2003.

[3] J. Richalet, A. Rault, J. L. Testud, and J. Papon, "Model predictive heuristic control," Automatica, vol. 14, no. 5, pp. 413-428, 1978.

[4] C. R. Cutler, and B. L. Ramaker, "Dynamic matrix control-A computer control algorithm," in Proc. joint automatic control conference, no. 17, p. $72,1980$.

[5] M. Sauvée, P. Poignet, E. Dombre, and E. Courtial, "Image based visual servoing through nonlinear model predictive control," in Proc. the 45th IEEE Conference on Decision and Control, San Diego, USA, 2006, IEEE, 2006, pp. 1776-1781.

[6] M. Sauvée, P. Poignet, and E. Dombre, "Ultrasound image-based visual servoing of a surgical instrument through nonlinear model predictive control," The International Journal of Robotics Research, vol. 27, no. 1, pp. 25-40, 2008

[7] G. Allibert, E. Courtial, and F. Chaumette, "Predictive control for constrained image-based visual servoing," IEEE Transactions on Robotics, vol. 26, no. 5, pp. 933-939, 2010.

[8] G. Allibert, E. Courtial, and F. Chaumette, "Visual servoing via nonlinear predictive control," in Visual Servoing via Advanced Numerical Methods, London: Springer, 2010, pp. 375-393.

[9] F. Chaumette, and S. Hutchinson, "Visual servo control. I. Basic approaches," IEEE Robotics \& Automation Magazine, vol. 13, no. 4, pp. 82-90, 2006.

[10] C. G. Economou, M. Morari, B. O. Palsson, 'Internal model control: Extension to nonlinear system," Industrial \& Engineering Chemistry Process Design and Development, vol. 25, no. 2, pp. 403-411, 1986.

[11] D. Q. Mayne, J. B. Rawlings, C. V. Rao, and P. O. M. Scokaert, "Constrained model predictive control: Stability and optimality," Automatica, vol. 36 , no. 6 , pp. 789-814, 2000. 